\title{
El juguete: una herramienta pedagógica en el juego grupal, para el nivel inicial
}

The toy: a pedagogical tool in the group play, for the initial level

1 Dayana Jazmín Sánchez Condo

https://orcid.org/0000-0002-1023-9045

Universidad Técnica de Ambato, Facultad de Ciencias Humanas y de la Educación,

Carrera de Educación Inicial, Tungurahua, Ambato, Ecuador,

dsanchez6646@uta.edu.ec

2 Irelys Sánchez Fernández

iD https://orcid.org/0000-0003-4075-6008.

Universidad Técnica de Ambato, Facultad de Ciencias Humanas y de la Educación,

Carrera de Educación Inicial, Ambato, Ecuador,

i.sanchez@uta.edu.ec

3 Gissela Alexandra Arroba López

https://orcid.org/0000-0002-7846-6535

Universidad Técnica de Ambato, Facultad de Ciencias Humanas y de la Educación,

Carrera de Educación Inicial, Ambato, Ecuador,

ga.arroba@uta.edu.ec

4 Tamara Yajaira Ballesteros Casco iD https://orcid.org/ 0000-0002-5962-7962

Universidad Técnica de Ambato, Facultad de Ciencias Humanas y de la Educación,

Carrera de Educación Inicial, Ambato, Ecuador,

ty.ballesteros@uta.edu.ec

Artículo de Investigación Científica y Tecnológica

Enviado: 24/12/2021

Revisado: $29 / 12 / 2021$

Aceptado: $12 / 01 / 2022$

Publicado:08/03/2023

DOI: https://doi.org/10.33262/concienciadigital.v6i1.4.1994

Cítese:

Sánchez Condo, D. J., Sánchez Fernández, I., Arroba López, G. A., \& Ballesteros Casco, T. Y. (2023). El juguete: una herramienta pedagógica en el juego grupal, para el nivel inicial.

ConcienciaDigital,

6(1.4),

211-232.

https://doi.org/10.33262/concienciadigital.v6i1.4.1994

CONCIENCIA DIGITAL, es una Revista Multidisciplinar, Trimestral, que se publicará en soporte electrónico tiene como misión contribuir a la formación de profesionales competentes con visión humanística y crítica que sean capaces de exponer sus resultados investigativos y científicos en la misma medida que se promueva mediante su intervención cambios positivos en la sociedad. https://concienciadigital.org

La revista es editada por la Editorial Ciencia Digital (Editorial de prestigio registrada en la Cámara Ecuatoriana de Libro con No de Afiliación 663) www.celibro.org.ec

Esta revista está protegida bajo una licencia Creative Commons Attribution Non Commercial No Derivatives

4.0 International. Copia de la licencia: http://creativecommons.org/licenses/by-nc-nd/4.0/ 


\section{Palabras}

claves: juguete, juego grupal, herramienta pedagógica, aprendizaje, socialización.

\section{Keywords:}

toy, group

game, pedagogical tool, learning, socialization

\section{Resumen}

Introducción. Este artículo se basa en una investigación realizada por Sánchez \& Sánchez en el año 2021, la cual permitió profundizar en la importancia del juguete en el juego grupal, para los niños del nivel Inicial; para esto se revisaron ampliamente referencias bibliográficas y se identificaron las características técnicas y pedagógicas del juguete. Objetivo. Proponer tipos de juguetes que permitan un óptimo aprendizaje en los niños de Educación Inicial. Metodología. El diseño de la investigación fue cualitativo por la naturaleza de los datos y el tipo de la información, con un nivel de profundidad Descriptivo, por esta razón la investigación es tipo Básica. Para la recolección de información se aplicaron las técnicas de Entrevista, Grupo focal y para el análisis de la información, la triangulación de datos; la población estuvo constituida por seis maestras de educación Inicial de la Unidad Educativa Teresa Flor y cinco psicólogos y docentes expertos en el tema, como unidad de análisis se ha considerado a cada persona. Resultados. Los principales aportes de la investigación se pueden resumir en lo siguiente: la mayoría de los autores indican que los juguetes facilitan la socialización del niño de mejor manera en los juegos grupales; el juguete es un instrumento pedagógico que estimula el aprendizaje con múltiples beneficios en cuanto a la actividad lúdica, mientras que el juego grupal se le considera como el lenguaje del niño. Conclusión. Se concluyó que los juguetes considerados más adecuados para los niños del nivel inicial I y II, de 3 a 5 años, son: títeres, rompecabezas, juegos de ensarte, instrumentos musicales, juegosde roles, muñecos, cuentos, legos, plastilina, entre otros, los que permiten que se desarrollen integralmente $\mathrm{y}$, al mismo tiempo, mejoren su capacidad de concentración y atención.

\section{Abstract}

Introduction. This article is based on research conducted by Sánchez \& Sánchez in the year 2021, that allowed to deepen the importance of the toy in group play at the initial level; for which the bibliographic references were extensively reviewed, and the technical and pedagogical characteristics of the toy were identified. Objective. To propose types of toys that allow optimal learning. Methodology. The research design was qualitative, due to the nature of the data and the type of information, with a 
descriptive depth level, for this reason the research is basic type. For the collection of information, the techniques of interview, focus group and forthe analysis of the information the triangulation of data were applied; The population is made up of six early childhood teachers from the Teresa Flor Educational Unit and five psychologists and teachers who are experts on the subject. Each person has been considered as the unit of analysis. Results. The main contributions of the research can be summarized as follows: most authors indicate that toys facilitate the child's socialization in a better way in group games; the toy is a pedagogical instrument that stimulates learning with multiple benefits in terms of playful activity, while group play is considered the child's language. Conclusion. The toys most suitable for children from initial level I and II, from 3 to 5 years old, are: puppets, puzzles, stringing games, musical instruments, role plays, dolls, stories, legos, plasticine, among others, which allow them to develop comprehensively and, at the same time, improve their capacity for concentration and attention.

\section{Introducción}

Desde épocas remotas, se da mucha importancia a aprender mientras se juega, filósofos clásicos como Platón y Aristóteles, animaban a los padres para que dieran a sus hijos juguetesque ayudaran a pensar y a desenvolverse para sus actividades futuras. Autores como Cuellar et al. (2018), concluyen que, a pesar de las precisiones conceptuales de los diferentes teóricos, todos concuerdan en la importancia del juego en el aspecto psicológico, pedagógico y social del ser humano.

Investigaciones descriptivas como la realizada por Duek (2016), tienen como objetivo describir teorías y metodologías relevantes sobre el uso del juego y proponer clasificaciones de los juegos infantiles, cualquier objeto que rodee la cotidianeidad infantil y les llame la atención por algún motivo, puede constituirse en un juguete. Concluye esta autora que el juegono es un juego, sino un espacio en el que se convergen la historia, educación y relaciones, sobre las cuales se organiza la cotidianeidad. Esta es la justificación para analizar este tema con mayor profundidad y relacionarlo a la luz de los tiempos contemporáneos.

Pastor et al. (2010), afirman que la forma en que se vinculan entre niños y adultos adquieren habilidades, conocimientos y destrezas que se ponenal descubierto mediante la conducta. 
A través del juego, las vivencias que tiene el niño con sus iguales ayudan en los aspectos sociales de su desarrollo, además, son elementos fundamentales para el proceso mediante el cual se descubre a sí mismo como individuo. Por lo tanto, la vida del niño con sus iguales tiene importancia desde un punto de vista afectivo ydel desarrollo de su concepto de sí mismo. (Linguido \& Zorraindo, 2014)

En un estudio realizado en México, titulado "La socialización a través del juego", se señala lo siguiente:

... el juego grupal es una excelente estrategia didáctica para trabajar las deficiencias de socialización en un grupo, ya que es una actividad placentera donde el niño se sientelibre para expresar sus sentimientos, para echar a volar su imaginación, donde puede olvidar su timidez, etc. En algunos casos podemos observar que en los juegos el niño refleja su estado de ánimo, incluso algunos problemas o acciones que pudieran ejercerhacia su persona (Alonso, 2015, pág. 25).

Además, les permitirá que la interacción se desarrolle y se fortalezca; debido a la importancia de estos se estimula el aprendizaje, el diálogo y la socialización. Por este motivo, es transcendental promover el estudio de las habilidades que realizaron los autores Valdez et al. (2019), mediante la aplicación del instrumento "Prueba de las habilidades de interacción social en niños de tres a seis años".

En el juego, los niños desarrollan y dominan su propio pensamiento tanto como sus estructuras. "El lenguaje oral se convierte en el medio del pensamiento porque refleja la operación intelectual. Esto significa que el pensamiento adquiere un carácter verbal y el lenguaje, un carácter intelectual" (Gonzáles, 2017). A estas conclusiones llegó el autor Vigotsky, psicólogo y pedagogo, luego de aplicar un juego grupal, desarrollado por él, entre un adulto y un grupo de 20 niños preescolares, en una institución educativa pública en la ciudad de Bogotá, Colombia.

Complementariamente Kac (2012), en su libro titulado "El periodo de iniciación en la Educación Infantil", comenta que es necesario construir herramientas teóricas y prácticas que sirvan de apoyo y faciliten la adaptación de los niños a la vida institucional y se transformen en aprendizaje útil y significativo, considera que es necesario elevar el nivel de aprendizaje mediante el diseño de planificaciones estratégicas que creen las condiciones paraque niños y sus entornos familiares puedan canalizar la ansiedad y la angustia que provocaenfrentarse a lago nuevo e incorporarse a la vida institucional como parte integrante de ella. Se concluye por lo tanto, que las actividades recreativas son excelentes para incentivar eldesarrollo de las habilidades competenciales de forma llamativa, motivacional y divertida,para quien las practican, a manera de ejemplo, estos investigadores aplicaron la técnica de Redes Semánticas Naturales (RSN) y para el análisis se usó la teoríade la percepción y el HJ-Biplot para 
recopilar la información, en un grupo de niños, niñas y jóvenes juarenses de 6 a 16 años, que participaron en el Campamento de Verano promovido por la Universidad Autónoma de Ciudad Juárez (UACJ), se observó el juego que los niñosdesarrollaron y como dominaron las estructuras de su propio pensamiento.

Asimismo, el lenguaje oral facilita la interacción social, así como el desarrollo de las representaciones simbólicas, esto se presenta porque los niños aprenden en sus interacciones sociales a expresarse adecuadamente. "Dicho aprendizaje se adquiere al compartir símbolos sociales, a partir de los cuales es posible considerar la perspectiva de los otros, de acuerdo conlas necesidades de la situación comunicativa" (Gonzáles, 2018).

De esta manera, se considera el juego como una actividad inherente al ser humano, a través de este, el niño aprende a relacionarse dentro del ámbito familiar, material, cultural y social; en este sentido, "el juego está estrechamente ligado al desarrollo social, los hace aprender a tomar acuerdos, a interrelacionarse, a integrarse al grupo, a compartir ideas y sentimientos" (Prado, 2008).

Un ejemplo que ilustra lo comentado es el planteado por Gómez (2018), cuando se juega un partido de futbol, primero hay que enterarse y aceptar las reglas que se deben cumplir, en la mitad del partido, el niño no se puede llevar el balón porque piense que es de él, ya sabe que el portero permanece cerca del arco, que el defensa ayuda al arquero a defender su área, y quesi comete una falta a otro, recibe una sanción, etc.; es necesario que comprenda que hay límites, que las otras personas cuentan, que hay que cumplir la ley, y en relación con esto la aceptación de reglas sociales es un modelo necesario que debe comprender y aceptar.

El juego social se entiende como aquella actividad en la que los niños interactúan entre sí y en diferentes grados, a medida que el niño va creciendo, su juego tiende a ser más social y cooperativo. A través del juego, el niño entra en relación con sus iguales y eso le permite conocer a las personas que lo rodean, además aprenden a cooperar, ayudar, compartir y solucionar problemas sociales (Ríos, 2013).

Para Ugalde (2011) "en un principio los niños juegan solos, después lo hacen al lado de otros niños, hasta que finalmente incluyen a los otros en su juego", en este punto el juego social seconsidera que ha evolucionado, ya que el niño incluye a los otros a sus juegos, esta es una evidencia de la disminución gradual del egocentrismo de cada niño.

En un estudio realizado por Molina (2008), se logra definir la cronología de las edades como las siguientes:

- Hasta los tres años no es sociable, sus actividades y conversaciones son en mayor partesolitarias, aún no se produce una interacción con otros niños 
- De 4 a 5 años, luego de haber hecho contacto con otros niños, se adapta a reuniones con mayor número de niños, en ese momento se ha encontrado a él y está en vías de aceptarse.

- A partir de los 5 años puede comprometerse con el grupo, abandona poco a poco su egocentrismo y empieza a identificar a otros para convertirlos en modelos e imitadores.

Para cuando finalice el nivel Inicial, los niños tienen bien identificadas las bases para mantenerrelaciones de grupo y hacer elecciones afectivas.

Para Sánchez \& Sánchez (2021), se ven con mucha frecuencia situaciones en las que cada niño asume su rol y manifiesta lo que siente, lo que posibilita una serie de aprestamientos sociales que lo preparan para la vida, una vez que se acople al tejido social, al terminar la adolescencia. Mediante el juego, el pequeño aprende a dar solución a problemas de su vida cotidiana, de una manera más significativa para su edad y lo más importante sin correr riesgos. El juego le permite al niño expresar sus emociones que en determinado momento lo pueden abrumar y angustiar.

Según Serrano (2017), para los docentes:

...el que los niños jueguen, es la oportunidad para que generen aprendizajes y logren sus propósitos. Es importante permitirles cierta libertad en su aula e institución, para que los niños realicen sus actividades de trabajo, mezclándolas con el juego, para que se impulsen y motiven a trabajar, se desenvuelvan y pierdan progresivamente el temor.De esta manera, asimilen la diversidad de reglas, para establecer espacios propios... (p.5)

Según Sánchez \& Sánchez (2021):

como herramienta pedagógica, el juego no puede faltar en las aulas de clase, en especial de educación inicial, ya que permite a los niños afianzar su seguridad y gracias a esto, socializar, de manera efectiva, en los juegos grupales con los demás. Los juegos deben considerarse como una actividad importante en el aula de clase, pues aportan una forma diferente de adquirir el aprendizaje, aportan descanso y recreación al niño.

En resumen, el niño necesita acción, manejar objetos, relacionarse con otros niños y esto se hace a través del juego, por ello la actividad lúdica es necesaria y vital para su desarrollo.

En relación con esto para Olivares (2015):

El juego es un instrumento trascendente de aprendizaje para la vida y por ello, es 
un importante instrumento en la educación; es necesario que se aproveche al máximo el potencial educativo del juego, por lo tanto, el docente debe intervenir haciendo uso deeste instrumento de manera eficaz, para permitir el crecimiento y desarrollo global de niños, mientras viven situaciones de placer y diversión. También debe buscar constituir una vía de aprendizaje del comportamiento cooperativo, propiciando situaciones de responsabilidad personal, solidaridad y respeto hacia los demás, se deben generar situaciones que supongan un reto, pero un reto superable y se debe evitar que en los juegos se destaquen siempre las mismas personas, diversificándolos y dando más importancia al proceso que al resultado final. (p.26)

Si bien hasta aquí se ha tratado el tema del juego, por otra parte, se hace necesario incorporar en este acápite una herramienta esencial que ayuda al buen desarrollo del niño/a: el "juguete",el cual tiene una influencia muy importante en el juego.

Autores como Fraser (1972) y Jaffé (2006), han contribuido en la recopilación de la historia de los juguetes y sus inventores, haciendo un extenso recorrido desde las cometas, las muñecas y las pelotas de las antiguas civilizaciones, hasta la moda de los tomagotchis, muñecos interactivos y robots. En la figura 1, se aprecia una línea de tiempo sobre la historia del juguete; historiadores calculan que ya desde la prehistoria los niños se divertían con juguetes, posiblemente elaborados de madera, arcilla o hueso; el juguete más antiguo que se ha localizado tiene cerca de unos 4.000 años (Sánchez \& Sánchez, 2021).

En Turquía se descubrió, en un yacimiento arqueológico, un sonajero hecho de terracota. Se conoce que los niños romanos y egipcios poseían estatuillas que personificaban a personas y animales, como los muñecos de hoy. En América, concretamente en Perú, los más pequeños jugaban con silbatos con forma de pájaros.

El autor Rubal (2017), concluye que "cualquier objeto puede convertirse en juguete cuando se usa la imaginación...”. 


\section{Figura 1}

Historia del juguete

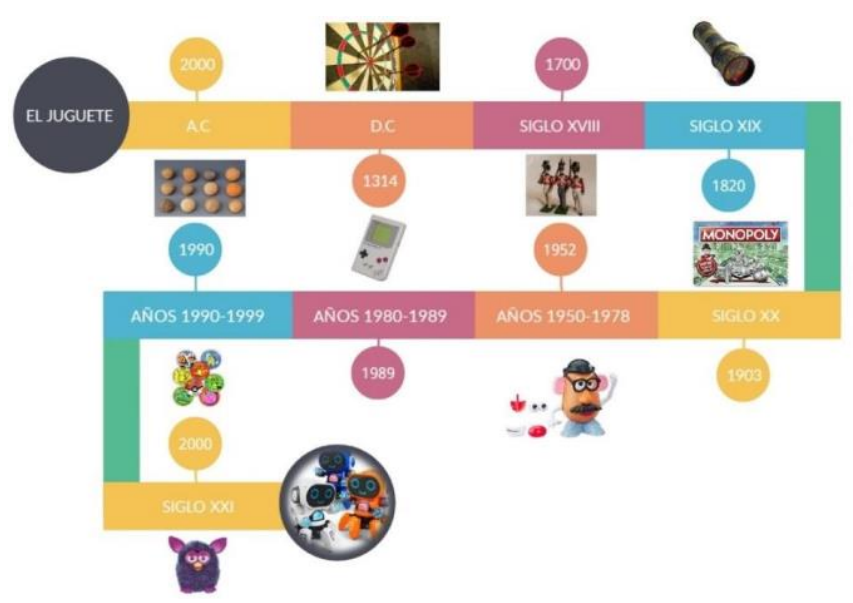

Nota: Los historiadores consideran que desde la prehistoria los niños se divertían con juguetesy que el más antiguo que se ha localizado tiene cerca de unos 4.000 años.

Fuente: Sánchez \& Sánchez (2021), el juguete en el juego grupal de los niños del nivel inicial.

En relación con esto, los juguetes son un medio imprescindible para que los niños alcancen su óptimo desarrollo, tiene un papel importante en la formación de los conceptos, aptitudes, expectativas y en la socialización de los niños (Torres et al., 2015). En el sector educativo los juguetes constituyen un útil instrumento de enseñanza aprendizaje, estimulan los sentidos para desarrollar habilidades, destrezas, formación de actitudes y valores, mediante el acceso a mayor y mejor información (Villanueva \& Rojas, 2019).

En este contexto, los docentes utilizan los juguetes como una herramienta pedagógica con el propósito de acompañar el aprendizaje de los niños y así potenciar su desarrollo. Existen muchas aplicaciones que se pueden usar como un juguete, como, por ejemplo: en el proceso de desarrollo de los niños, en las habilidades que deben fomentar, en las experiencias que viven con sus docentes en el aula y con sus familias en sus casas.

En este sentido, es importante tener en cuenta que el docente debe apropiarse de diversas herramientas que ayuden al desenvolvimiento de los niños, de una forma natural y espontánea, no reproduciendo aprendizajes, permitiéndoles adquirir conocimientos esenciales para su educación (Llerena et al., 2020).

Para finalizar en el trabajo de grado realizado por Guanoluisa (2015), se aclara que:

Los juguetes no pueden nunca verse limitados al sexo del niño, ni en modo alguno deben estimular cualidades negativas como: intolerancia, agresividad y violencia; en este sentido, el juego y los juguetes permiten educar el carácter del 
niño y estimular en él valores deseables como: altruismo, igualdad, compañerismo, capacidad de cooperación y tolerancia. (pág. 16)

Según Sánchez \& Sánchez (2021), en este sentido el juguete desempeña un papel importanteen la formación de conceptos, aptitudes, expectativas, y la socialización de los más pequeños. Desde siempre, los niños/as han tenido la necesidad de una actividad: moverse, curiosear, manipular objetos, crear, relacionarse, intercambiar acciones, vivencias y sentimientos.

Los juguetes son objetos que acompañan al niño y lo apoyan en el transcurso de su desarrollo y pese a que se hayan visto afectados por los adelantos tecnológicos, la intención sigue siendola misma: divertir, provocar alegría, generar satisfacción, motivar a la creación, dando la oportunidad de perfeccionar el aprendizajes y progreso en los niños (Barrera et al., 2015).

En relación con esto, para describir la importancia de los juguetes en el desarrollo infantil. Delgado (2016), propone una serie de beneficios para dar respuesta a la siguiente pregunta “¿por qué son tan importantes los juguetes en el desarrollo infantil?

- Estimulan el aprendizaje infantil, permitiendo que el niño descubra la existencia dediferentes formas, colores, tamaños y texturas.

- Desarrollan las habilidades motoras gruesas y finas, potenciando también la capacidadviso espacial.

- Permiten que el niño descubra las funciones de los objetos que le rodean, comprendiendo para qué se utiliza cada uno.

- Ayudan al pequeño a mejorar su equilibrio y coordinación, a la vez que estimulan elcontrol muscular.

- Facilitan la maduración de tres funciones mentales básicas: la asimilación, la comprensión y la adaptación a la realidad.

- Fomentan la creatividad y la imaginación, sobre todo cuando se trata de juguetes no convencionales como puede ser una caja de cartón, una cinta o unas simples botellas.

- Estimulan la autoconfianza infantil pues mientras el niño juega, siente que es el protagonista de la actividad, sabe que es capaz de dominar la tarea y los juguetes queutiliza.

- Mejoran el control de las emociones, a la vez que ayudan al pequeño a descubrir nuevas sensaciones y sentimientos.

- Favorecen la socialización y la creación de vínculos emocionales con las personas, yasea con otros niños o con los adultos.

- Potencian la comunicación y la capacidad de expresión infantil pues mediante 
los juguetes el niño puede expresar lo que piensa, siente o quiere hacer.

- Ayudan a lidiar con los problemas de la vida cotidiana, facilitando la resolución de conflictos, ya que el niño modela a través del juego tanto sus preocupaciones como lasposibles soluciones."

Morón (2010), propone una clasificación de los juguetes, como se muestra en la figura 2, ya que algunos tienden apromover un juego de mayor calidad que otros, este autor comenta que la mayoría de los juguetes que más se venden en la actualidad, corresponden a la categoría de los muy estructurados, que están estrechamente vinculados con los medios de comunicación.

Para Sánchez \& Sánchez (2021):

el juguete tiene un fuerte componente simbólico que facilitael desarrollo de la imaginación en los niños; pero también depende de la persona que juega con él y de su posición dentro del juego. En cualquier caso, la motivación más profunda del juego infantil es la lúdica, aunque también se puede tener en cuenta la capacidad de aprendizaje y relación social que permite.

\section{Figura 2}

Clasificación de los juguetes

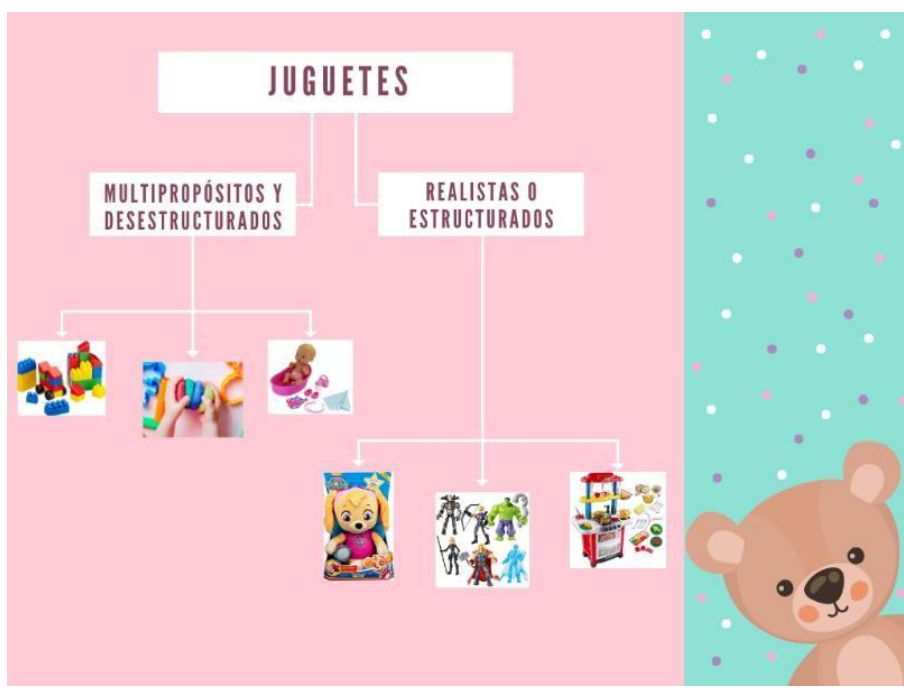

Nota: La mayoría de los juguetes que más se venden en la actualidad, corresponden a la categoría de los muy estructurados, muy vinculados con los medios de comunicación.

Fuente: Morón (2010). Un principio de intervención educativa: El juego y los juguetes en educación infantil.

Tal vez uno de los hechos más relevantes que el autor Antoñanzas (2005), destaca, sobre el uso del juguete, es que permite realizar al niño las primeras acciones mutuas con otros niños y con los adultos, con el paso del tiempo, este intercambio constituye un intermediario que losprepara para manejar ciertas situaciones de la vida social sin correr 
riesgos.

Otros hechos relevantes a destacar sobre el uso del juguete se ofrecen a continuación:

- Ayuda a transmitir valores simbólicos.

- Permite aprender características del medio social y cultural que le rodea.

- Ayuda en su desarrollo físico y mental.

- Estimulan el desarrollo de las facultades sensoriales, motrices, creativas e imaginativas.

- Ayuda a aceptar las limitaciones del mundo.

- Controlar su conducta al pasar de los sueños a la realidad.

- Ayuda para que la fantasía y la imaginación dominen su vida.

A partir de los estudios de Fiedrich Fröbel y Karl Gras, la función didáctica es consideradacomo clave para la pedagogía moderna, en relación con esto, el juego es una actividad que ayuda a preparar a los niños para su vida adulta, mientras que el juguete motiva a los niños a realizar actividades artísticas y creativas (Antoñanzas, 2005).

Para Glanzer (2006), el vínculo que se establece entre el niño y el juguete aporta lo siguiente:

- Un valor funcional que otorga sus alternativas de aprovechamiento.

- Un valor afectivo debido a que recibe emociones y sentimientos.

- Un valor cognitivo al apropiarse de nuevos conceptos y experiencias.

- Un valor pedagógico debido a que se convierte en objeto lúdico mediador del juego.Clasificación funcional de los juguetes:

- Afectivos, son receptores de sentimientos.

- Didácticos, permiten el desarrollo intelectual y cognitivo.

- Constructivos, estimula la percepción y la orientación espacial.

- Artísticos, permiten desarrollar la imaginación y la creatividad.

Desde otro punto de vista acerca de esta temática, se puede afirmar que, al combinar el juguetecon el juego grupal, se transforma en un material didáctico, útil para las docentes del nivel Inicial, ya que permite desarrollar las clases de forma espontánea y divertida, los niños aprenden a compartir con los demás, experiencias, reglas y por lo tanto socializan de una manera más efectiva. Además de los beneficios pedagógicos antes mencionados, el uso del juguete también contribuye a fortalecer la seguridad, control de las emociones y sentimientos, establecer vínculos afectivos con los demás, es una forma de expresión, y así como estos múltiples beneficios también ayudan al desarrollo físico y mental (Sánchez \& Sánchez, 2021).

"De 3 a 6 años es el turno de los juegos de imitación, de ensayo, de autosuficiencia, de despliegue del poder personal, de transformación de objetos y situaciones, de apropiación del medio ambiente, de interacción social y convivencia" (Rodríguez \& Ketchum, 2000). 
Aquí surge una interrogante importante: ¿qué tipo de juguete es el adecuado para utilizar? Para Blázquez-Blázquez y Benavente (2019), la elección de los juguetes debe realizarse teniendo en cuenta la seguridad para los niños, es decir, los juguetes deben reunir algunas características mínimas, para que animen al niño a estar activo, promuevan el juego intergeneracional y aviven su imaginación. Las principales características de los juguetes pueden ser: la creatividad, el diseño, que sea didáctico, educativo, seguro, que permita divertirse, funcional, que no inciten la violencia, que no sean peligrosos o tóxicos y que realmente sean significativos para su desarrollo (Herrara, 2015).

Morón (2010), en su estudio denominado "Un principio de intervención educativa: El juego yel juguete en la educación infantil" describe algunas características técnicas y pedagógicas del juguete orientadas hacia la: seguridad, duración, grado de realidad y estructuración, salubridade higiene, estética y adecuación a la edad, así como hacia las habilidades físicas, destrezas sociales, intereses y preferencias, inteligencia emocional y solución de problemas.

\section{Tabla 1}

Características técnicas y pedagógicas del juego

\begin{tabular}{|c|c|}
\hline Técnicas & Pedagógicas \\
\hline $\begin{array}{l}\text { Habilidades físicas: agarrar, sujetar, correr, } \\
\text { trepar, balancearse. }\end{array}$ & - Su cuerpo: habilidades, limitaciones \\
\hline $\begin{array}{l}\text { - Habla y lenguaje: desde el balbuceo, hasta } \\
\text { contar cuentos y chistes. }\end{array}$ & - Su personalidad: intereses, \\
\hline $\begin{array}{l}\text { Destrezas sociales: cooperar, negociar, } \\
\text { competir, seguir reglas, esperar turnos. }\end{array}$ & $\begin{array}{l}\text { Otras personas: expectativas,reacciones, cómo } \\
\text { llevarse con adultos y con niños/as. }\end{array}$ \\
\hline $\begin{array}{l}\text { - Inteligencia racional: comparar, categorizar, } \\
\text { contar, memorizar. }\end{array}$ & $\begin{array}{l}\text { El medio ambiente: explorar posibilidades, } \\
\text { reconocer peligros y límites. }\end{array}$ \\
\hline $\begin{array}{l}\text { - Inteligencia emocional: autoestima, compartir } \\
\text { sentimientos con otros. }\end{array}$ & $\begin{array}{l}\text { La sociedad y la cultura: roles, tradiciones, } \\
\text { valores. }\end{array}$ \\
\hline $\begin{array}{l}\text { - El juego ayuda a utilizar energía física y } \\
\text { mental de maneras productivas y/o } \\
\text { entretenidas. }\end{array}$ & $\begin{array}{l}\text { Dominio propio: esperar, perseverar, lidiar con } \\
\text { contra tiempos y derrotas. }\end{array}$ \\
\hline $\begin{array}{l}\text { - El juego enriquece la creatividad y la } \\
\text { imaginación }\end{array}$ & $\begin{array}{l}\text { - Solución de problemas: considerar e } \\
\text { implementar estrategias. }\end{array}$ \\
\hline - El juego estimula todos los sentidos. & $\begin{array}{l}\text { Toma de decisiones: reconocer opciones, } \\
\text { escoger, y lidiar con las consecuencias. }\end{array}$ \\
\hline
\end{tabular}

Fuente: Morón (2010) 
De esta manera, se aprecia como el juguete y el juego grupal según la literatura consultada, tienen un vínculo estrecho, los cuales en su conjunto ayudan al desarrollo integral de los niños/as de educación Inicial.

\section{Metodología}

Esta investigación utiliza un paradigma c rítico que permite descubrir y comprender elfenómeno de estudio. Como un aspecto clave, se selecciona un enfoque por la naturaleza de los datos y el tipo de la información, cualitativo, dado que analiza la importanciadel juego en el aspecto pedagógico.

Según Quintana (2006): "El investigador cualitativo depende de la información que logre recolectar en la observación de campo o en las entrevistas exploratorias para definir el foco del problema estudiado" (p.56).

Complementariamente, se considera un tipo de investigación Básica, por lo tanto, tiene como propósito la obtención de conocimientos sin tener en cuenta su aplicabilidad (Muntané, 2010). Al analizar el nivel de profundidad, se selecciona el tipo descriptivo, debido a que "tiene como objetivo describir algunas características fundamentales de conjuntos homogéneos de fenómenos, utilizando criterios sistemáticos que permiten establecer la estructura o el comportamiento de los fenómenos en estudio, proporcionando información sistémica y comparable con las otras fuentes" (Guevara et al., 2020).

La población estuvo constituida por un conjunto de individuos, objetos o eventos que tienen características comunes, en este caso, seis maestras de Educación Inicial de la Unidad Educativa Teresa Flor y cinco expertos; la muestra representa un subconjunto de la poblaciónen la que la elección de los elementos no depende de la probabilidad sino de las característicasde la investigación (Hernández et al., 2014); para esta investigación la muestra representa el $100 \%$ de la población. En estudios como el realizado por Espinoza \& Toscano (2015), comentan que se pueden utilizar técnicas, como la observación sistemática, la observación participante, encuestas, entrevistas, test, etc. para realizar estudios descriptivos.

Para obtener la información necesaria que permitió alcanzar los objetivos planteados en la investigación, se aplicó como técnicas de recolección de información la entrevista, que es un diálogo entre el entrevistador y el entrevistado, sobre un tema previamente determinado, y el grupo focal, que consiste en reunir a un grupo de participantes para discutir un tema de investigación determinado (Martínez, 2012); los instrumentos que se emplean es un guion de preguntas abiertas que dan la oportunidad para que los participantes se expresen libremente, recopilando detalles importantes para la investigación y para el grupo focal, preguntas previamente estructuradas. 
Complementariamente, se utiliza la triangulación de datos, que se refiere al uso de diferentesestrategias y fuentes de información sobre la recolección de datos que permite contrastar la información recabada (Aguilar \& Barroso, 2015).

\section{Resultados}

La aplicación de técnica de la entrevista y del grupo focal, mediante preguntas abiertas, permitió recabar información de las docentes de la "Unidad Educativa Teresa Flor" y de los expertos que participaron en el grupo focal, para obtener los diferentes puntos de vista sobre el tema de la presente investigación, además, se retuvo en cuenta para el análisis de los resultados la información obtenida de las diferentes fuentes bibliográficas revisadas.

Al tener la presente investigación un enfoque cualitativo, el procesamiento de la información se obtuvo aplicando la técnica de triangulación, mediante el análisis de datos de manera independiente por cada uno de los docentes y expertos participantes y, posteriormente, se sometió estos análisis a comparación por categorías de las variables, dependientes e independientes. Al final, los hallazgos encontrados en la triangulación serán el resultado del consenso de las respuestas dadas por los profesionales participantes y por los autores analizados en las fuentes bibliográficas.

Los resultados más relevantes obtenidos, estuvieron dirigidos hacia que, en las primeras etapasde formación de los niños, es necesario estimular la exploración y descubrimiento ordenado, para que se facilite su desenvolvimiento en el futuro. Los esfuerzos de los docentes estarán orientados a crear experiencias de aprendizaje significativas, para captar su atención y estimular los órganos sensoriales. Para lograr este propósito, es recomendable utilizar un conjunto variado de materiales para realizar juegos didácticos. El juego permite generar y acumular experiencias para facilitar el aprendizaje y orientar al niño a crear sus propios conocimientos, en este sentido, la herramienta pedagógica con orientación lúdica que facilita la interacción es el juguete, pues proporciona el desarrollo sensorial y emocional del niño. Eneste sentido, es importante tener en cuenta el rango de edad, para alcanzar un óptimo desarrollosocial y de los conceptos iniciales.

Para cumplir con uno de los objetivos propuestos en la investigación, se realizó una amplia revisión de sitios web, que enseñan a los padres a elegir y a comprar el mejor juguete según la edad de su hijo, así como también páginas que comercializan juguetes para niños.

Para ello, se muestra en la tabla 2 el tipo de juguete y la frecuencia con la que es recomendadosu uso, esta información se utilizó para proponer aquellos que son más adecuados para el desarrollo físico y cognitivo. 


\section{DDigital}

Tabla 2

Juguetes recomendados

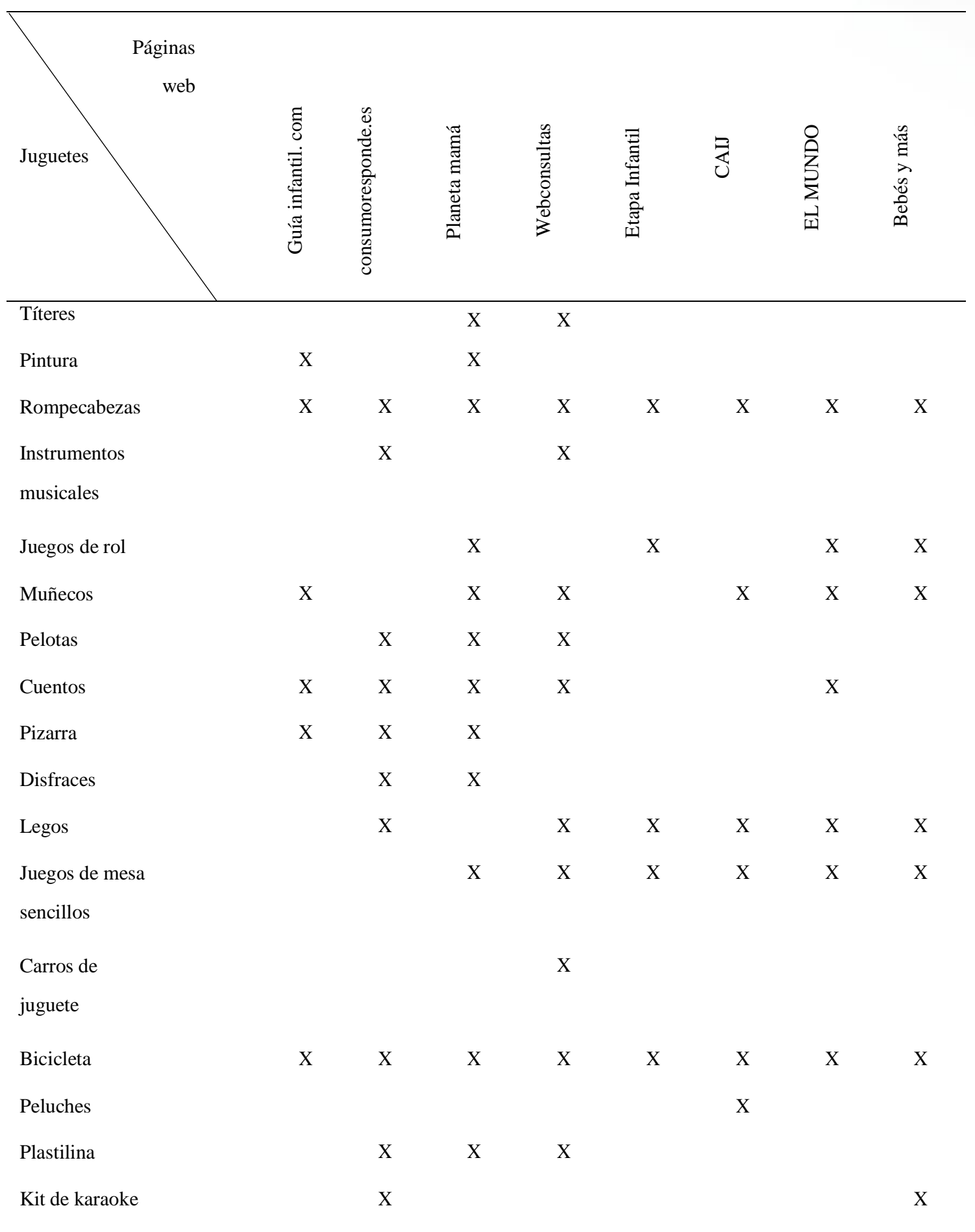

Fuente: Sánchez \& Sánchez (2021) 


\section{DDigital}

Seguidamente, en la figura 3 se aprecian los tipos de juguetes más adecuados según la edad, para el juego grupal de los niños del nivel inicial.

\section{Figura 3}

Juguetes recomendados para niños de 3 a 5 años

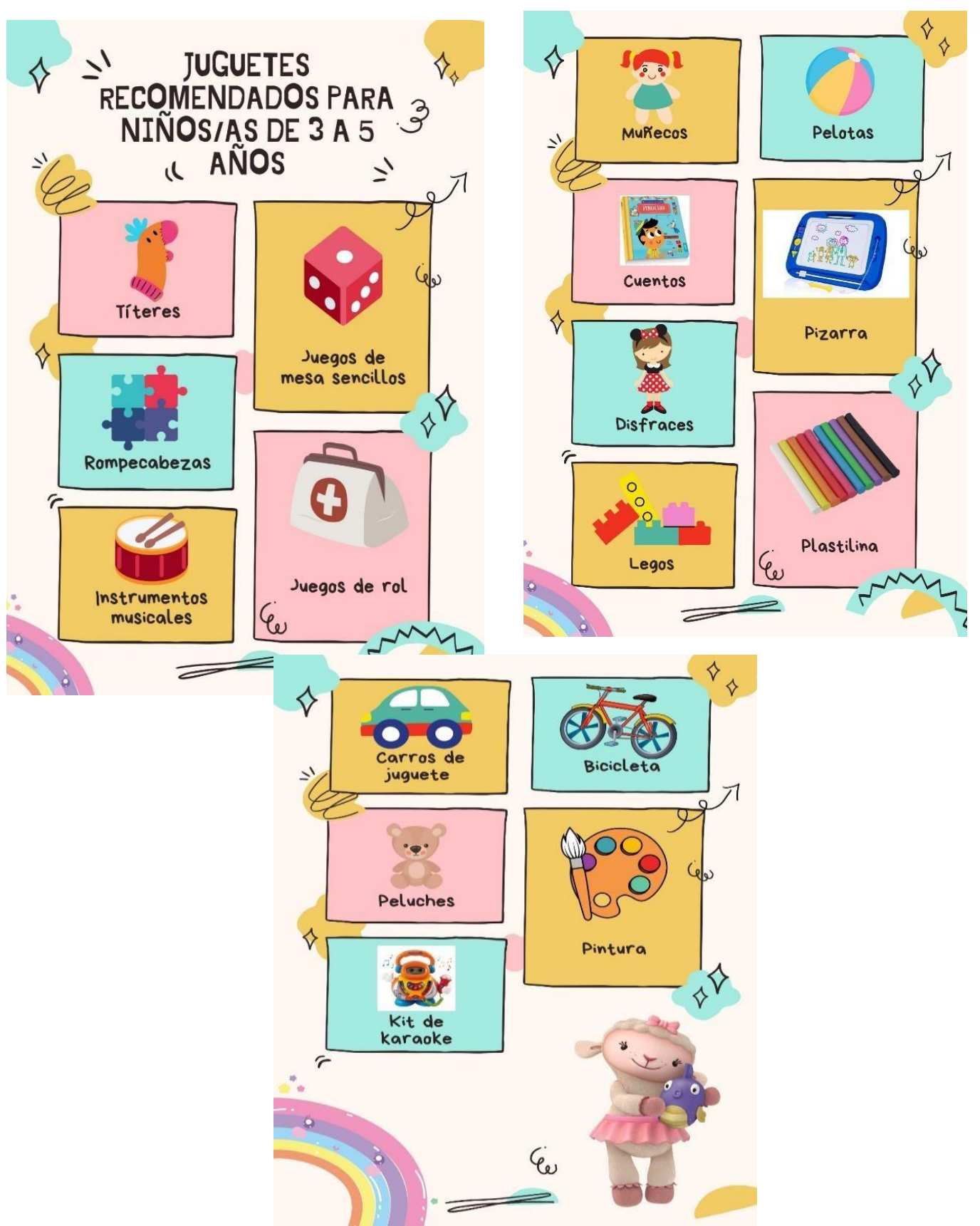

Nota: Los juguetes más recomendados implican un desarrollo integral para los niños de 3 a 5 años. Fuente: Sánchez \& Sánchez (2021). El juguete en el juego grupal de los niños del nivel inicial. 
Sumado a los tipos de juguetes anteriormente recomendados, tenemos que la socialización delniño se logra mediante la aplicación de una estrategia didáctica que le ayuda a interactuar con otros niños, aprendiendo a compartir y al mismo tiempo identificando reglas de comportamiento social. Este conjunto de experiencias le permite conocerse a sí mismo y a tener un fuerte sentido de identidad. Un medio para lograr estos objetivos es la aplicación deuna serie de actividades recreativas para promover el desarrollo de sus habilidades sociales y su autoestima, potenciando el sentido de pertenencia al grupo. Es especialmente el juego grupal el que favorece el aprendizaje gradual, mediante la personificación de roles, la aplicación de reglas y conductas sociales, finalmente la utilización del juguete facilita que las actividades compartidas sean más beneficiosas para su desarrollo, porque ayudan a los niños a explorar la realidad, a encontrar nuevos caminos, desarrollar su imaginación, probar estrategias, aprender, acertar, fallar, equivocarse y volverlo a intentar.

Después del análisis de información y de los resultados obtenidos el presente estudio defiendela idea en torno a la pregunta de investigación ¿Qué papel cumple el juguete en el juego grupal?

Para comprender cuál es el papel del juguete en el juego grupal, se debe argumentar primeramente que el juguete es un medio importante en el aula de nivel Inicial, porque permiteaprender de una manera más significativa y de la misma manera, juega un papel fundamentalen el juego grupal, ya que consigue que la socialización entre sus pares sea más fácil, así comopermitir establecer un diálogo debido a la curiosidad que generan los juguetes.

Además, se hace referencia a los autores Torres et al. (2015), quienes plantean que "los juguetes son un medio indispensable para que el niño logresu óptimo desarrollo, además de que juega un papel importante en la formación de los conceptos, aptitudes, expectativas y socialización en los niños."

\section{Conclusiones}

- A partir de la bibliografía revisada se pudo comprobar que desde tiempos muy antiguos se ha utilizado el juguete como medio para la educación, compilando evidencias literarias sobre el uso del juguete en el juego grupal, lo que permitió concluir que la mayoría de los autores, coinciden en señalar que los juguetes facilitan la socialización delniño de mejor manera en los juegos grupales

- La revisión literaria fue fundamental para describir las características técnicas y pedagógicas del juguete, concluyendo que es un instrumento pedagógico que estimulael aprendizaje con múltiples beneficios, en cuanto a 
la actividad lúdica, mientras que el juego grupal se considera como el lenguaje del niño, facilita la interacción social y afianza su autoestima. En este sentido, la importancia de utilizar la metodología del juego - trabajo, para desarrollar el bienestar emocional, social, físico y cognitivo en el nivel inicial.

- Por último, se considera que algunos juguetes adecuados para los niños del nivel Inicial I y II, es decir, de 3 a 5 años, son: títeres, rompecabezas, juegos de ensarte,instrumentos musicales, juegos de roles, muñecos, cuentos, legos, plastilina, entre otros, pues permiten el desarrollo integral y, al mismo tiempo, mejoran su capacidad de concentración y atención.

\section{Referencias bibliográficas}

Aguilar Gavira, S., \& Barroso Osuna, J. (2015). La triangulación de datos como estrategia eninvestigación educativa. Revista de Medios y Educación.

Alonso Castro, E. (mayo de 2015). Universidad pedagógica nacional. Universidad pedagógica nacional: https://d1wqtxts1xzle7.cloudfront.net/56828059/103ALONSOCASTRO.pdf?1 52946 $1546=\&$ response-contentisposition=inline\%3B+filename\%3DLA_SOCIALIZACION_A_TRAVES_D EL_JUEGO_Pre.pdf \&Expires $=1620860694 \&$ Signature $=J H 0 o 2 F \sim S c a A 5 f 6 H V$ B4UXj0fe4--CYIcFCJH8GI5RPPgZ79b3hDS1Y

Antoñanzas Mejía, F. (2005). Artistas y juguetes. Dialnet. España. Universidad Complutense Madrid: https://eprints.ucm.es/id/eprint/7124/1/T28496.pdf

Barrera Cardozo, L., Perdomo Ordoñez, B., Serrato Serrato, M., Trujillo Hernández, Y. \& González González, M. (2015). Impacto de los juguetes en los procesos formativos delos niños. Plumilla educativa.

Blázquez-Blázquez, E. \& Benavente, R. (2019). ¿Son seguros los juguetes que compramos? La importancia del control aduanero en el sector del juguete. Revista de Plásticos Modernos, 9-14.

Cuellar Cartaya, M. E., Tenreyro Mauriz, M. \& Castellón León, G. (2018). El juego en la educación preescolar. Fundamentos históricos. SciELO.

Delgado, J. (14 de noviembre de 2016). Etapa Infantil. Etapa Infantil: https://www.etapainfantil.com/juguetes-desarrollo-ninos

Duek, C. (2016). El juego infantil contemporáneo: medios de comunicación, nuevas 
prácticasy clasificaciones. SciELO.

Espinoza, E. \& Toscano, D. (2015). Metodología de la Investigación Educativa y Técnica. Machala: Universidad Técnica de Machala.

Fraser, A. (1972). A history of toys. New York: Spring Books.

Glanzer, M. (01 de noviembre de 2006).

EspacioLogopédico.com. espacioLogopédico.com: https://www.espaciologopedico.com/revista/articulo/1250/los-juguetesmaterial- ludico-didactico-autonomo-parte-ii.html

Gómez Ramírez, J. F. (2018). El juego infantil y su importancia en el desarrollo. México.

Gonzáles, C. (2017). Actividad comunicativa interactiva entre un adulto y un grupo de niñospreescolares en una experiencia de juego grupal. Dialnet.

Gonzáles, C. (2018). Actividad comunicativa interactiva en una experiencia de juego grupal con niños en edad preescolar. Cuadernos Hispanoamericanos de Psicología, 45.

Guanoluisa, D. (2015). Facultad de Ciencia y Tecnología. Facultad de Ciencia y Tecnología. (Tesis de Licenciatura). Universidad Técnica del Norte. http://repositorio.utn.edu.ec/bitstream/123456789/5788/1/05\%20FECYT\%2029 77\% 20TRABAJO\%20DE\%20GRADO.pdf

Guevara, G., Verdesoto, A. \& Castro, N. (2020). Metodologías de investigación educativa. Revista científica mundo de la investigación y el conocimiento.

Hernández Sampieri, R., Fernández, C., \& Baptista, M. d. (2014). Metodología de la investigación. México: McGraw-Hill.

Herrara Tamayo, N. (2015). Tendencias y factores de influencia en el consumo de juguetes ydispositivos tecnológicos. Escuela de ingeniería de Antioquia.

Jaffé, D. (2006). The history of toys: from spinning tops to robots. England: Stroud.

Kac, M. (2012). Período de iniciación en la Educación Infantil. Rosario: Novedades Educativas.

Linguido, M., \& Zorraindo, M. (2014). Proceso de socialización en la etapa preescolar. Revista de psicología.

Llerena, A., Sánchez, I. \& Hernández, Y. (2020). La retroalimentación y su influencia 
en la consolidación de aprendizajes en proceso, de niños de Educación Inicial. Conciencia Digital, 60-75.

Martínez, N. (2012). Reseña metodológica sobre los grupos focales. Diálogos.

Molina Prieto, R. (2008). El juego como medio de socialización. Innovación y experiencias educativas.

Morón Macías, C. (2010). Un principio de intervención educativa: El juego y los juguetes eneducación infantil. Temas para la educación.

Muntané, J. (2010). Introducción a la investigación básica. RAPD.

Olivares Cardoza, S. (marzo de 2015). Universidad de Piura. Universidad de Piura: https://pirhua.udep.edu.pe/bitstream/handle/11042/2674/EDUC_033.pdf?sequ ence $=1$

Pastor Fasquelle, R., Nashiki Angulo, R. \& Pérez Figueroa, M. (2010). El desarrollo y aprendizaje infantil, y su observación. México: Puentes para crecer.

Prado Bello, K. (diciembre de 2008). Universidad Pedagógica Nacional. Universidad Pedagógica Nacional: http://200.23.113.51/pdf/27185.pdf

Quintana, A. (2006). Metodología de Investigación Científica Cualitativa. Lima: UNMSM. Ramos, C. (2017). Los paradigmas de la investigación científica. UNIFÉ.

Ríos, M. (2013). Universidad Internacional de la Rioja. Universidad Internacional de la Rioja: https://reunir.unir.net/bitstream/handle/123456789/1910/2013_01_31_TFM_E STUD IO_DEL_TRABAJO.pdf?sequence $=1 \&$ isAllowed $=\mathrm{y}$

Rodríguez, M. \& Ketchum, M. (2000). Creatividad en los juegos juguetes. México: México.

Rubal, M. (24 de noviembre de 2017). La vanguardia. La vanguardia: https://www.lavanguardia.com/vida/juniorreport/20171124/433060886059/historia- juguete-infancia-imaginacion.html

Sánchez, D. \& Sánchez, I. (28 de septiembre de 2021). El juguete en el juego grupal de los niños del nivel inicial. Repositorio UTA: https://repositorio.uta.edu.ec/jspui/handle/123456789/33319

Serrano, Á. (2017). El juego en preescolar: acción compleja y perversa. Congreso nacional de investigación educativa. 
Torres Ramos, Y., Ramos Villena, V. \& Tortoló Fernández, S. (2015). Los juguetes como medio de desarrollo del niño de la primera infancia. Atenas.

Ugalde Galindo, M. (diciembre de 2011). Universidad Pedagógica Nacional. Universidad Pedagógica Nacional: http://200.23.113.51/pdf/28333.pdf

Valdez Ruiz, J., Valenzuela Robles, V., \& Ayala Madrid, G. (2019). La interacción social como medio para favorecer las habilidades sociales de menores preescolares durante la sesión de educación física, en un jardín de niños urbano. CONISEN.

Villanueva Quispe, C. \& Rojas Alavi, N. (2019). Importancia del material didáctico en el aula de educación inicial en familia comunitaria. Importancia del material didáctico en el aula de educación inicial en familia comunitaria: https://es.scribd.com/document/440744899/articulo

\section{Ciencia Ligital}


El artículo que se publica es de exclusiva responsabilidad de los autores y no necesariamente reflejan el pensamiento de la Revista Conciencia Digital.

\section{Ciencia}

El artículo queda en propiedad de la revista y, por tanto, su publicación parcial y/o total en otro medio tiene que ser autorizado por el director de la Revista Conciencia Digital.
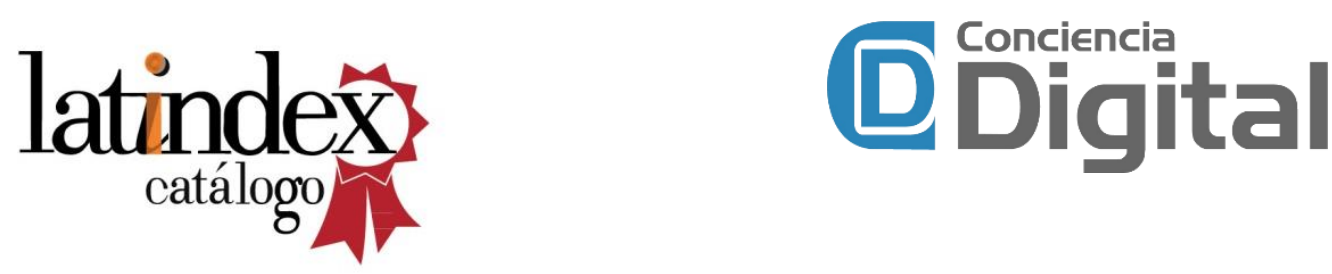

Indexaciones

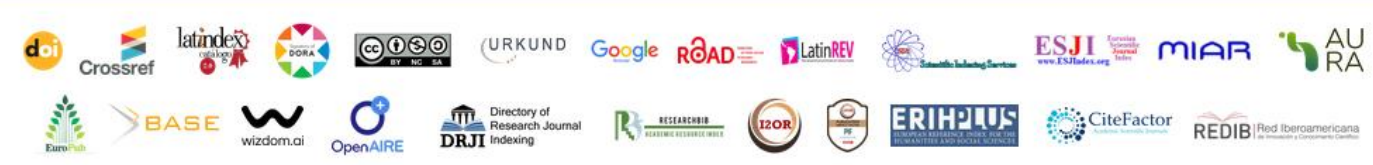

